\title{
Robust Beamforming in Cognitive Radio
}

\author{
Gan Zheng ${ }^{\S}$, Shaodan $\mathrm{Ma}^{\ddagger}$, Kai-Kit Wong ${ }^{\S}$, and Tung-Sang $\mathrm{Ng}^{\ddagger}$ \\ $\S$ Adastral Park Research Campus, University College London \\ Martlesham Heath, IP5 3RE, UK, Email: \{g.zheng,k.wong\}@ adastral.ucl.ac.uk \\ ‡Department of Electrical and Electronic Engineering, The University of Hong Kong \\ Pokfulam, Hong Kong, Email: \{sdma,tsng\}@eee.hku.hk
}

\begin{abstract}
In cognitive radio, it is crucial to control the interference from secondary users (SUs) to primary users (PUs). This paper studies the use of transmit beamforming in the cognitive secondary network for enhancing the performance of a SU while controlling the interference to the PUs. In particular, we propose to maximize the service probability of the SU with a number of probability constraints on the interference level at the PUs with the aid of imperfect channel state information (CSI). Modeling the CSI uncertainty as an additive Gaussian noise, it is shown that the optimum can be realized by second-order cone-programming (SOCP) in tandem with a one-dimensional search. Results reveal that the proposed approach provides a technique to tradeoff the performance between the PUs and the SU, making an analytical connection between non-robust and worst-case systems.
\end{abstract}

\section{INTRODUCTION}

Radio spectrum is a precious resource for wireless communications. According to federal communications commission (FCC) [1], spectrum utilization depends very much on place and time and most spectrum is under-utilized. Cognitive radio (CR), first proposed in [2], is a new paradigm for exploiting the spectrum resources in a dynamic way [3], [4].

In CR, the spectrum is shared by the primary users (PUs) and the secondary users (SUs). The PUs are the licensed users who have the primary rights to access the spectrum while the SUs can occupy the spectrum only if they do not interrupt the communication of PUs. In practice, the SUs will sense the spectrum to detect any PUs' activities by using, for instance, the likelihood ratio test [5], energy detection [6], matchedfiltering-based method [7] and cyclostationary detection [8], etc, and decide if a SU is permitted for reusing the spectrum.

While detection of PU is an important and difficult task for using the spectrum holes in CR [9], spectrum sharing for the PUs and SUs is also possible if the interference caused by the SUs is properly controlled [10]. In this paper, we shall focus on the case in which the PU and SU coexist and in particular investigate the use of transmit beamforming at the secondary CR network for interference control with the aid of imperfect channel state information (CSI) at the SU transmitter.

The problem under investigation is related to robust optimization against channel mismatches, which is usually tackled by two approaches: i) worst-case optimization and ii) stochastic or statistical analysis. In worst-case approaches, the CSI error is considered to be bounded and the system is required to maintain a given quality-of-service (QoS) for every possible channel realizations and error conditions [11]. This technique is, nonetheless, unsuitable if the CSI error is unbounded, for instance, in the case when the CSI is estimated from training, which results in an unbounded Gaussian uncertainty in the channel estimates. For this reason, statistical approaches have emerged as an attractive means to provide robustness, in the form of confidence level measured by probability [12].

In this paper, we consider a CR network with a number of PUs and one SU, and aim to maximize the service probability of the SU while controlling the interference levels to the PUs based on some preset probability constraints. The construction of the problem facilitates the robust solution to tradeoff the performance between the PUs and the SU in CR that bridges a non-robust secondary system and the worst-case robust SU solution. In particular, we show that the optimal beamforming solution can be obtained by second-order cone-programming (SOCP) and a one-dimensional search.

In the sequel, we shall use the following notations. Vectors are column vectors and denoted in lower case bold $\mathbf{x}$ while matrices are upper case bold $\mathbf{A}$. The superscripts ${ }^{\dagger}$ stands the conjugate transposition. $|\cdot|$ takes the modulus of a complex number and $\|\cdot\|$ returns the Frobenius norm of a vector or matrix. $\mathbf{x} \sim \mathcal{C N}(\mathbf{m}, \mathbf{V})$ means that $\mathbf{x}$ is a vector of complex Gaussian random variables and has a mean vector of $\mathbf{m}$ with a covariance matrix of $\mathbf{V}$.

\section{System Model And Problem Statement}

Consider a CR network with $L(\geq 1)$ PUs and one SU. The transmitter of the SU has $N$ antennas while there is only one antenna at the SU receiver and each of the PUs. The channels between the SU transmitter and the PUs are denoted by $\left\{\mathbf{g}_{l}\right\}$ for $l=1,2, \ldots, L$ and we use $\mathbf{h}$ to denote the channel between the SU transmitter and receiver. In $\mathrm{CR}$, the problem of interest is to maximize the SU's received power for a given transmit power constraint $P$ while controlling the interferences on the PUs to certain acceptable levels, say $\left\{I_{l}\right\}$. With a beamforming vector $\mathbf{w}$ at the SU transmitter, it is written as

$$
\begin{array}{ll}
\max _{\mathbf{w}} & \left|\mathbf{h}^{\dagger} \mathbf{w}\right|^{2} \\
\text { s.t. } & \left\{\begin{array}{c}
\|\mathbf{w}\|^{2} \leq P \\
\left|\mathbf{g}_{l}^{\dagger} \mathbf{w}\right|^{2} \leq I_{l}, \forall l .
\end{array}\right.
\end{array}
$$

This problem is nonconvex but can be reformulated to the 
following convex and more specifically, SOCP problem:

$$
\begin{aligned}
& \max _{\mathbf{w}} \mathbf{h}^{\dagger} \mathbf{w} \\
& \text { s.t. } \quad\left\{\begin{aligned}
\|\mathbf{w}\|^{2} & \leq P \\
\operatorname{Im}\left\{\mathbf{h}^{\dagger} \mathbf{w}\right\} & =0 \\
\left|\mathbf{g}_{l}^{\dagger} \mathbf{w}\right|^{2} & \leq I_{l}, \forall l,
\end{aligned}\right.
\end{aligned}
$$

which, as a result, can be optimally solved if CSI is known perfectly at both the SU transmitter and receiver.

While in practice, the CSI available to the SU transmitter is destined to be imperfect, due to estimation errors or other factors such as quantization. In particular, in this paper, we model these errors as additive Gaussian so that

$$
\left\{\begin{aligned}
\mathbf{h} & =\hat{\mathbf{h}}+\Delta \mathbf{h} \\
\mathbf{g}_{l} & =\hat{\mathbf{g}}_{l}+\Delta \mathbf{g}_{l}, \forall l
\end{aligned}\right.
$$

where $\hat{\mathbf{h}}$ and $\left\{\hat{\mathbf{g}}_{l}\right\}$ denote the channel estimates known at the transmitter, and $\Delta \mathbf{h}$ and $\left\{\Delta \mathbf{g}_{l}\right\}$ are the respective CSI errors, which are further modeled as [12]

$$
\left\{\begin{aligned}
\Delta \mathbf{h} & \sim \mathcal{C N}\left(\mathbf{0}, \sigma_{h}^{2} \mathbf{I}\right), \\
\Delta \mathbf{g}_{l} & \sim \mathcal{C N}\left(\mathbf{0}, \sigma_{l}^{2} \mathbf{I}\right),
\end{aligned}\right.
$$

with the variances $\sigma_{h}^{2}$ and $\left\{\sigma_{l}^{2}\right\}$ indicating the CSI quality.

Given this model, the optimization problem becomes

$$
\begin{gathered}
\max _{\mathbf{w}} \operatorname{Prob}\left(\left|\mathbf{h}^{\dagger} \mathbf{w}\right|^{2} \geq \gamma\right) \\
\text { s.t. }\left\{\begin{array}{c}
\|\mathbf{w}\|^{2} \leq P \\
\operatorname{Prob}\left(\left|\mathbf{g}_{l}^{\dagger} \mathbf{w}\right|^{2} \leq I_{l}\right) \geq \varepsilon_{l}, \forall l,
\end{array}\right.
\end{gathered}
$$

where the probabilistic measures are done over the CSI error statistics. Note that the optimization is performed to maximize the service probability of the SU defined in accordance with a target signal-to-interference plus noise ratio (SINR) threshold, $\gamma$, and the interferences are controlled probabilistically at some predetermined levels, $\left\{\varepsilon_{l}\right\}$. The parameters $\left\{\varepsilon_{l}\right\}$ can be used to tradeoff the performance between the PUs and the SU.

To proceed, we express the service probability by noting

$$
y=\left|\mathbf{h}^{\dagger} \mathbf{w}\right|^{2}=\left|\hat{\mathbf{h}}^{\dagger} \mathbf{w}+\Delta \mathbf{h}^{\dagger} \mathbf{w}\right|^{2},
$$

which is recognized as a non-central Chi-square random variable with degrees of freedom $n=2$, variance $\sigma_{y}^{2}=\frac{\|\mathbf{w}\|^{2} \sigma_{h}^{2}}{2}$ and noncentrality parameter $s_{y}^{2}=\left|\hat{\mathbf{h}}^{\dagger} \mathbf{w}\right|^{2}$. As such,

$$
\operatorname{Prob}\left(\left|\mathbf{h}^{\dagger} \mathbf{w}\right|^{2} \geq \gamma\right)=Q_{1}\left(\frac{s_{y}}{\sigma_{y}}, \frac{\sqrt{\gamma}}{\sigma_{y}}\right),
$$

where $Q_{1}$ is the generalized Marcum's Q function. Similarly, we can also express the interference probability constraints in $Q_{1}$. As a consequence, we have

$$
\begin{aligned}
\max _{\mathbf{w}} & Q_{1}\left(\frac{\left|\hat{\mathbf{h}}^{\dagger} \mathbf{w}\right|}{\sqrt{\frac{\|\mathbf{w}\|^{2} \sigma_{h}^{2}}{2}}}, \frac{\sqrt{\gamma}}{\sqrt{\frac{\|\mathbf{w}\|^{2} \sigma_{h}^{2}}{2}}}\right) \\
\text { s.t. } & \left\{\begin{aligned}
\|\mathbf{w}\|^{2} \leq P \\
Q_{1}\left(\frac{\left|\hat{\mathbf{g}}_{l}^{\dagger} \mathbf{w}\right|}{\sqrt{\frac{\|\mathbf{w}\|^{2} \sigma_{l}^{2}}{2}}}, \frac{\sqrt{I_{l}}}{\sqrt{\frac{\|\mathbf{w}\|^{2} \sigma_{l}^{2}}{2}}}\right) \leq 1-\varepsilon_{l}, \forall l .
\end{aligned}\right.
\end{aligned}
$$

The rest of the paper will be devoted to finding the optimal transmit beamforming solution of (8) for CR.

\section{The Optimal Solution}

To solve (8), we observe that it involves the generalized Marcum's Q function in both the objective function and the constraints, which depend on the beamforming vector $\mathrm{w}$. Due to the interference constraints, in general, it is anticipated that the SU's transmit power will not reach its maximum, $P$, and this is another reason that makes it difficult to deal with.

A closer observation reveals that $\|\mathbf{w}\|$ can be treated as a single parameter that affects the system performance. In what follows, we rewrite (8) as

$$
\begin{aligned}
\max _{\mathbf{w}} & Q_{1}\left(\frac{\left|\hat{\mathbf{h}}^{\dagger} \mathbf{w}\right|}{\sqrt{\frac{\|\mathbf{w}\|^{2} \sigma_{h}^{2}}{2}}}, \frac{\sqrt{\gamma}}{\sqrt{\frac{\|\mathbf{w}\|^{2} \sigma_{h}^{2}}{2}}}\right) \\
\text { s.t. } & \left\{\begin{array}{l}
\|\mathbf{w}\|^{2} \leq P \\
\left|\hat{\mathbf{g}}_{l}^{\dagger} \mathbf{w}\right| \leq Q_{1}^{-1}\left(\frac{\sqrt{I_{l}}}{\sqrt{\frac{\|\mathbf{w}\|^{2} \sigma_{l}^{2}}{2}}}, 1-\varepsilon_{l}\right) \sqrt{\frac{\|\mathbf{w}\|^{2} \sigma_{l}^{2}}{2}}, \forall l,
\end{array}\right.
\end{aligned}
$$

where $Q_{1}^{-1}$ is the inverse function to find the first parameter, given the second parameter and the probability, i.e.,

$$
Q_{1}\left(Q_{1}^{-1}(b, a), b\right)=a .
$$

This has motivated us to first solve the problem for a given transmit power $\|\mathbf{w}\|^{2}=p$ and then search the optimal $p$ to obtain the global optimal solution. Importantly, if $\|\mathbf{w}\|^{2}=p$ is fixed, then (8) is an SOCP and can be solved efficiently and optimally. The proposed method is described in detail next.

\section{A. Proposed Algorithm}

The proposed algorithm is a loop and is expected to identify the optimum after convergence. Define the resolution $\Delta P \triangleq$ $\frac{P}{N}$ for some large $N$ and a feasible set $\mathcal{S}=\emptyset$.

1) Initialize the iteration index $i=1$.

2) If $i \leq N$, set $p_{i}=i \Delta P$ and solve the following SOCP

$$
\begin{array}{ll}
\max _{\mathbf{w}} & \left|\hat{\mathbf{h}}^{\dagger} \mathbf{w}\right| \\
\text { s.t. } & \left\{\begin{array}{c}
\|\mathbf{w}\|^{2} \leq p_{i} \\
\left|\hat{\mathbf{g}}_{l}^{\dagger} \mathbf{w}\right|^{2} \leq f\left(I_{l}, \varepsilon_{l}, p_{i}, \sigma_{l}\right), \forall l,
\end{array}\right.
\end{array}
$$

where

$$
f\left(I_{l}, \varepsilon_{l}, p_{i}, \sigma_{l}\right) \triangleq Q_{1}^{-1}\left(\frac{\sqrt{I_{l}}}{\sqrt{\frac{p_{i} \sigma_{l}^{2}}{2}}}, 1-\varepsilon_{l}\right) \sqrt{\frac{p_{i} \sigma_{l}^{2}}{2}} .
$$

If the problem is feasible and the obtained solution $\mathbf{w}$ satisfies $\|\mathbf{w}\|^{2}=p_{i}$, then update $\mathcal{S}:=\mathcal{S} \bigcup\{\mathbf{w}\}$.

Also, update $i:=i+1$ and if $i>N$, go to Step 3); otherwise, go back to Step 2).

3) The optimal solution can be obtained by

$$
\mathbf{w}_{\mathrm{opt}}=\arg \max _{\mathbf{w} \in \mathcal{S}} Q_{1}\left(\frac{\left|\hat{\mathbf{h}}^{\dagger} \mathbf{w}\right|}{\sqrt{\frac{\|\mathbf{w}\|^{2} \sigma_{h}^{2}}{2}}}, \frac{\sqrt{\gamma}}{\sqrt{\frac{\|\mathbf{w}\|^{2} \sigma_{h}^{2}}{2}}}\right) .
$$




\section{B. Optimality Analysis}

Theorem 1: The proposed algorithm is optimal.

Proof: If we replace the " $\leq$ " in the power constraint of (11) with an equality sign, obviously, the proposed algorithm achieves the optimal solution. We now argue that even with " $\leq$ ", the proposed algorithm can still find the optimum. The advantage of (11) is that it makes the problem solvable while with "=", the problem will become intractable.

It is possible that for some $p_{i}$, the obtained beamforming solution $\mathbf{w}\left(p_{i}\right)$ is indeed optimal but $\left\|\mathbf{w}\left(p_{i}\right)\right\|^{2}<p_{i}$. However, the proposed algorithm will not miss any possible optimal solutions, because it goes through all possible $p_{i} \leq P$.

\section{THE BENCHMARK: WORST-CASE APPROACH}

An alternative, but more conservative, robust design method is based on the worst-case criterion if the CSI uncertainties are bounded, which can be formulated as

$$
\begin{aligned}
\max _{\mathbf{w}} & \min _{\|\Delta \mathbf{h}\| \leq \xi^{(h)}}\left|\mathbf{h}^{\dagger} \mathbf{w}\right|^{2} \\
\text { s.t. } & \left\{\begin{array}{c}
\|\mathbf{w}\|^{2} \leq P \\
\max _{\left\|\Delta \mathbf{g}_{l}\right\| \leq \xi_{l}^{(g)}}\left|\mathbf{g}_{l}^{\dagger} \mathbf{w}\right|^{2} \leq I_{l}, \forall l .
\end{array}\right.
\end{aligned}
$$

Note that it is possible to use an ellipsoidal region to bound the CSI errors and the principle is the same. Further, (14) in its current form is not convex, but can be reformulated to an SOCP problem as follows:

$$
\begin{aligned}
\max _{\mathbf{w}} & \hat{\mathbf{h}}^{\dagger} \mathbf{w}-\xi^{(h)}\|\mathbf{w}\| \\
\text { s.t. } & \left\{\begin{array}{l}
\|\mathbf{w}\|^{2} \leq P \\
\left|\hat{\mathbf{g}}_{l}^{\dagger} \mathbf{w}\right| \leq \sqrt{I_{l}}-\xi_{l}^{(g)}\|\mathbf{w}\|, \forall l .
\end{array}\right.
\end{aligned}
$$

To have a fair comparison between the proposed algorithm and the above worst-case based approach, we find the bounds $\xi^{(h)}$ and $\left\{\xi_{l}^{(g)}\right\}$ such that they are bounded with probabilities

$$
\left\{\begin{array}{l}
\operatorname{Prob}\left(\|\Delta \mathbf{h}\|^{2} \leq\left(\xi^{(h)}\right)^{2}\right)=\delta \text { for some } \delta>0, \\
\operatorname{Prob}\left(\left\|\Delta \mathbf{g}_{l}\right\|^{2} \leq\left(\xi_{l}^{(g)}\right)^{2}\right)=\varepsilon_{l}, \forall l
\end{array}\right.
$$

It is interesting to see the similarity between the last constraint in (15) and that in (9). In (9), the right-hand-side

$$
Q_{1}^{-1}\left(\frac{\sqrt{I_{l}}}{\sqrt{\frac{\|\mathbf{w}\|^{2} \sigma_{l}^{2}}{2}}}, 1-\varepsilon_{l}\right) \sqrt{\frac{\|\mathbf{w}\|^{2} \sigma_{l}^{2}}{2}}
$$

is a complicated function in $I_{l}, \sigma_{l}^{2}$ and $\|\mathbf{w}\|^{2}$, while in (15), it takes the simple form of $\sqrt{I_{l}}-\xi_{l}^{(g)}\|\mathbf{w}\|$.

\section{Simulation Results}

Simulations are conducted to evaluate the performance of the proposed system in independent and identically distributed (i.i.d.) Rayleigh flat-fading channels, i.e., $\mathbf{g}_{l} \sim \mathcal{C N}(0, \mathbf{I}), \forall l$, and $\mathbf{h} \sim \mathcal{C N}(0, \mathbf{I})$. The noise at each PU and the SU is also assumed to be zero-mean and unit-variance complex Gaussian. In addition, all channel error variances are assumed to be 0.05 , i.e., $\sigma_{h}^{2}=\sigma_{l}^{2}=0.05, \forall l$. The interference levels to the PUs are required to be $90 \%$ acceptable, or $\varepsilon_{l}=0.9, \forall l$. The maximum transmitted signal-to-noise ratio (SNR) for the SU, defined as $\frac{P}{N_{0}}$, is set to be $10(\mathrm{~dB})$. The received SNR for each PU has a similar definition. We also assume that the SU transmitter has three antennas and the PU transmitter has two antennas serving two PUs, i.e., $N=3$ and $L=2$. Results for the following schemes are compared: i) the proposed algorithm, ii) the worst-case method, and iii) the non-robust method.

In Fig. 1, we provide the results for the cumulative distribution function (CDF) of the received interference power at PU 1 from the SU when the interference temperature is set to $0(\mathrm{~dB})$, i.e., $\frac{I_{l}}{N_{0}}=0(\mathrm{~dB})$ for $l=1,2$. As can be seen, the resulting interference from the proposed algorithm is lower than $0(\mathrm{~dB})$ with a probability of $90 \%$, while the worst-case method ensures that the interference power never exceeds 0 (dB). On the other hand, the non-robust method does not take into account the possible CSI and as a result, more than $60 \%$ of the time, the interference level is over $0(\mathrm{~dB})$.

The effect of interference control on the bit-error-rate (BER) for PU 1 is shown in Fig. 2 assuming $\frac{I_{l}}{N_{0}}=0(\mathrm{~dB})$ for $l=$ 1,2 . To produce the results, for the PU network, we use zeroforcing beamforming in [13] so that no inter-user interference is present within the PU network. It is observed that the nonrobust technique results in very poor performance due to the ignorance of CSI errors. Considerable performance gain can be obtained using the proposed algorithm and the worst-case based approach in all received SNR regions. In particular, the worst-case approach achieves much lower BER than others.

Fig. 3 demonstrates the CDF results of the received signal power at the SU. It is observed that the non-robust method outputs the strongest signal because most interference constraints are violated while the signal power of the worst-case approach is the weakest because it focuses on the interference control on every possible channel estimation error.

The effect on the BER of the SU against different interference temperature requirements ranging from $\frac{I_{l}}{N_{0}}=-12 \sim 0$ (dB), for $l=1,2$, is plotted in Fig. 4. It is seen that the BER for all schemes decreases if more interference can be tolerated. Also, the worst-case approach sacrifices to gain absolute control of interference and has the worst BER performance while the non-robust method achieves the best BER performance at the cost of no control on interference to the PUs. The result coincides with that in Fig. 3. We have seen that the proposed algorithm provides a good tradeoff between the control of interference and the enhancement of the desired signal.

\section{CONCLUSION}

This paper has studied the stochastic robust transmit beamforming in CR to balance the interference control for PUs and signal enhancement for SU using probabilistic constraints. It has been shown that the problem of interest can be optimally solved using SOCP in tandem with a one-dimensional search. Simulation results have also illustrated the obtained robustness against the non-robust method and the improved performance tradeoff compared with the worst-case based robust design. 


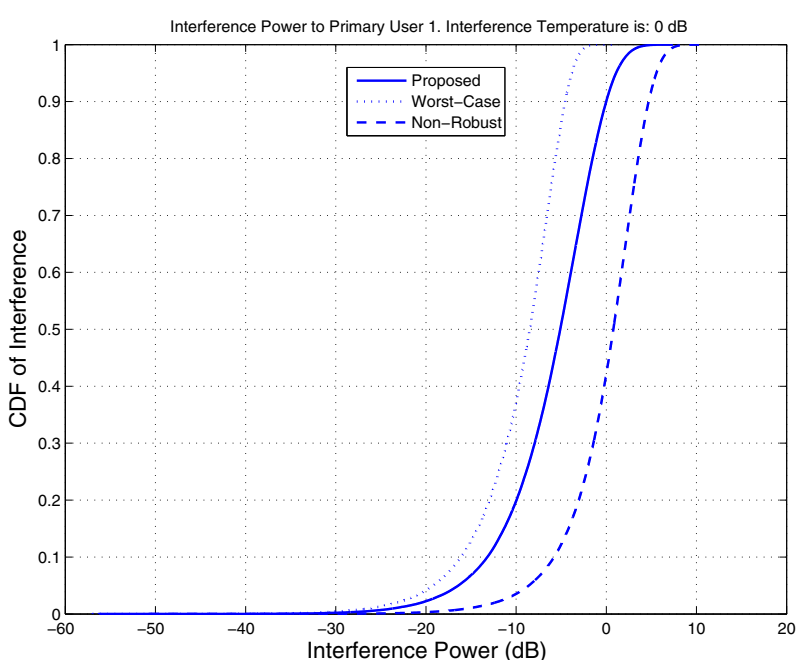

Fig. 1. The CDFs of the received interference power at PU 1 with $\frac{I_{l}}{N_{0}}=0$ (dB) for $l=1,2$.

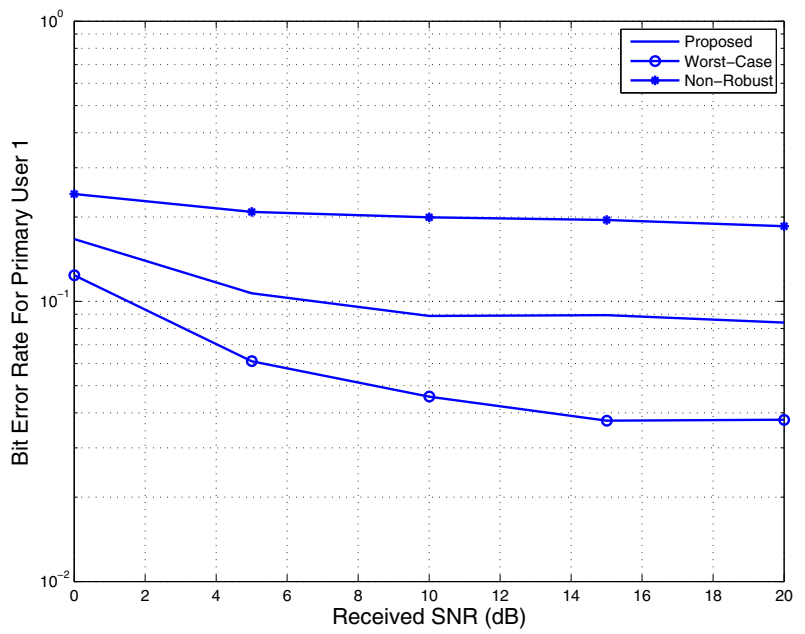

Fig. 2. The BERs of PU 1 for various received SNR $\frac{P}{N_{0}}$ with $\frac{I_{l}}{N_{0}}=0$ (dB) for $l=1,2$.

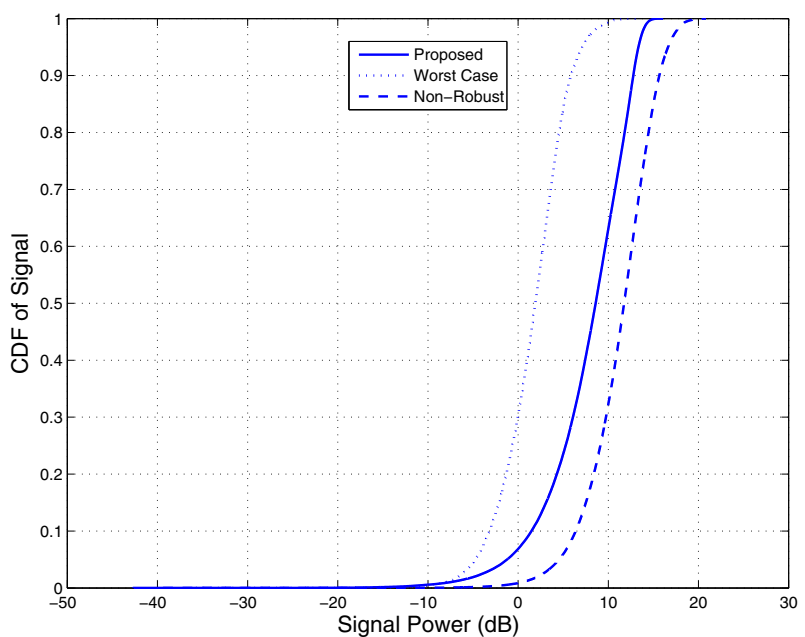

Fig. 3. The CDFs of the received signal power at the SU with $\frac{I_{l}}{N_{0}}=0(\mathrm{~dB})$ for $l=1,2$.

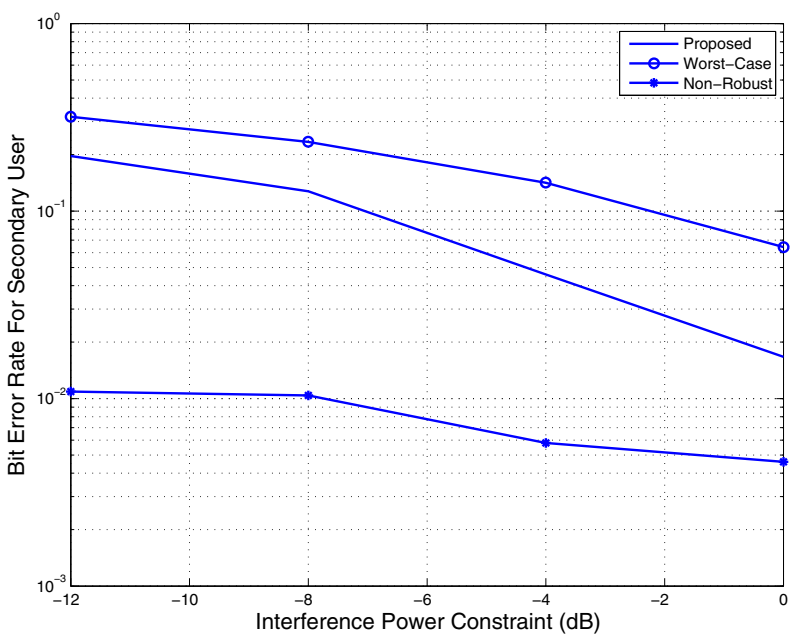

Fig. 4. The BERs of the SU for various interference requirements $\frac{I_{l}}{N_{0}}$.

\section{REFERENCES}

[1] "Spectrum policy task force," Federal Communications Commission, ET Docket No. 02-135, Tech. Rep., Nov. 2002.

[2] J. Mitola and G. Q. Maguire, "Cognitive radio: Making software radios more personal," IEEE Pers. Commun., vol. 6, no. 6, pp. 13-18, Aug. 1999.

[3] S. Haykin, "Cognitive radio: Brain-empowered wireless communications," IEEE J. Select. Areas Commun., vol. 23, no. 2, pp. 201-220, Feb. 2005.

[4] S. M. Mishra, A. Sahai, and R. W. Brodensen, "Cooperative sensing among cognitive radios," in Proc. IEEE Int. Conf. Commun., Jun. 2006.

[5] S. M. Kay, Fundamentals of Statistical Signal Processing: Detection Theory, vol. 2. Prentice Hall, 1998.

[6] R. Tandra and A. Sahai, " Fundamental limits on detection in low SNR under noise uncertainty," in Proc. WirelessCom 2005, Jun. 2005.

[7] D. Cabric, A. Tkachenko, and R. W. Brodersen, "Spectrum sensing measurements of pilot, energy, and collaborative detection," in Proc. Military Comm. Conf., pp. 1-7, Oct. 2006.

[8] W. A. Gardner, "Exploitation of spectral redundancy in cyclostationary signals," IEEE Sig. Proc. Mag., vol. 8, pp. 14-36, 1991.

[9] Y. C. Liang, Y. Zeng, E. C. Y. Peh, and A. T. Hoang, "Sensingthroughput tradeoff for cognitive radio networks," IEEE Trans. Wireless Commun., vol. 7, no. 4, pp. 1326-1337, Apr. 2008.

[10] A. Ghasemi and E. S. Sousa,"Fundamental limits of spectrum-sharing in fading environments," IEEE Trans. Wireless Commun., vol. 6, no. 2, pp. 649-658, Feb. 2007.

[11] H. Cox, R. M. Zeskind, and M. M. Owen, "Robust adaptive beamforming," IEEE Trans. Sig. Proc., vol. 35, no. 10, pp. 1365-1376, Oct. 1987.

[12] K. L. Bell, Y. Ephraim, and H. L. V. Trees, "A bayesian approach to robust adaptive beamforming," IEEE Trans. Sig. Proc., vol. 48, no. 2, pp. 386-398, Feb. 1987.

[13] Z. G. Pan, K. K. Wong, and T. S. Ng, "Generalized multiuser orthogonal space division multiplexing," IEEE Trans. Wireless Commun., vol. 3, no 6, pp. 1-5, Nov. 2004. 